\title{
Article \\ Structural Analysis of a Genetically Encoded FRET Biosensor by SAXS and MD Simulations
}

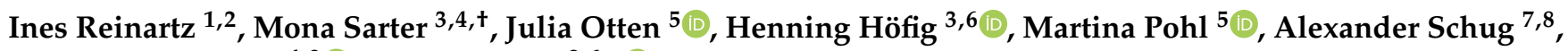 \\ Andreas M. Stadler ${ }^{4,9}$ (D) and Jörg Fitter ${ }^{3,6, *(1)}$
}

1 Institute for Automation and Applied Informatics, Karlsruhe Institute of Technology, Hermann-von-Helmholtz-Platz 1, 76344 Eggenstein-Leopoldshafen, Germany; ines.reinartz@kit.edu

2 HIDSS4Health-Helmholtz Information and Data Science School for Health, 76344 Eggenstein-Leopoldshafen, Germany

3 I Physikalisches Institut (IA), AG Biophysik, RWTH Aachen University, 52074 Aachen, Germany; mona.sarter@stfc.ac.uk (M.S.); h.hoefig@fz-juelich.de (H.H.)

4 Forschungszentrum Jülich, IBI-8/JCNS-1, 52428 Jülich, Germany; a.stadler@fz-juelich.de

5 Forschungszentrum Jülich, IBG-1, 52426 Jülich, Germany; j.otten@fz-juelich.de (J.O.); ma.pohl@fz-juelich.de (M.P.)

6 Forschungszentrum Jülich, IBI-6, 52428 Jülich, Germany

7 John von Neumann Institute for Computing, Jülich Supercomputing Centre, Forschungszentrum Jülich, 52428 Jülich, Germany; al.schug@fz-juelich.de

8 Faculty of Biology, University of Duisburg-Essen, 45141 Essen, Germany

9 Institut für Physikalische Chemie, RWTH Aachen University, 52074 Aachen, Germany

* Correspondence: fitter@physik.rwth-aachen.de; Tel.: +49-241-80-27209

+ Present address: ISIS Pulsed Neutron and Muon Facility, Science and Technology Facilities Council, Rutherford Appleton Laboratory, Harwell Science and Innovation Campus, Oxon OX11 0QX, UK.

Citation: Reinartz, I.; Sarter, M.; Otten, J.; Höfig, H.; Pohl, M.; Schug, A.; Stadler, A.M.; Fitter, J. Structural Analysis of a Genetically Encoded FRET Biosensor by SAXS and MD Simulations. Sensors 2021, 21, 4144. https://doi.org/10.3390/s21124144

Academic Editors: Stefano Bettati, Stefano Bruno, Luca Ronda and Marialaura Marchetti

Received: 14 May 2021

Accepted: 11 June 2021

Published: 16 June 2021

Publisher's Note: MDPI stays neutral with regard to jurisdictional claims in published maps and institutional affiliations.

Copyright: (c) 2021 by the authors. Licensee MDPI, Basel, Switzerland. This article is an open access article distributed under the terms and conditions of the Creative Commons Attribution (CC BY) license (https:/ / creativecommons.org/licenses/by/ $4.0 /)$.

\begin{abstract}
Inspired by the modular architecture of natural signaling proteins, ligand binding proteins are equipped with two fluorescent proteins (FPs) in order to obtain Förster resonance energy transfer (FRET)-based biosensors. Here, we investigated a glucose sensor where the donor and acceptor FPs were attached to a glucose binding protein using a variety of different linker sequences. For three resulting sensor constructs the corresponding glucose induced conformational changes were measured by small angle X-ray scattering (SAXS) and compared to recently published single molecule FRET results (Höfig et al., ACS Sensors, 2018). For one construct which exhibits a high change in energy transfer and a large change of the radius of gyration upon ligand binding, we performed coarse-grained molecular dynamics simulations for the ligand-free and the ligand-bound state. Our analysis indicates that a carefully designed attachment of the donor FP is crucial for the proper transfer of the glucose induced conformational change of the glucose binding protein into a well pronounced FRET signal change as measured in this sensor construct. Since the other FP (acceptor) does not experience such a glucose induced alteration, it becomes apparent that only one of the FPs needs to have a well-adjusted attachment to the glucose binding protein.
\end{abstract}

Keywords: glucose sensor; green fluorescence protein (GFP); single-molecule FRET; small angle $\mathrm{X}$-ray scattering (SAXS); coarse-grained molecular dynamics (MD)

\section{Introduction}

Important representatives of biosensors are molecular structures that link highly specific ligand-binding properties of biological macromolecules, mainly proteins, to a physical observable, which can be quantified by a read-out measurement [1-3]. In particular, periplasmic binding proteins (PBPs) offer a large potential, since they provide an allosteric linkage between binding of an effector ligand and a pronounced large-scale hinge-bending movement of two globular domains [4,5]. In the case of genetically encoded Förster resonance energy transfer (FRET)-based sensors, the sensors are often constructed as 
fusions of full-length protein chromophores, typically green fluorescent protein variants of different colors, attached via short linkers to the ligand binding protein termini. Based on known crystal structures of several PBPs, the distance in the range of $4-5 \mathrm{~nm}$ between $\mathrm{N}$ and $\mathrm{C}$ termini is well suited to enable Förster resonance energy transfer (FRET) between the two attached fluorescent proteins (FPs) acting as a donor/acceptor pair [5,6]. Importantly, the FRET transfer efficiency can vary significantly upon small changes in distance or orientation between the fluorescent proteins, caused by the ligand-induced hinge-bending movement of the ligand binding protein. Resulting changes in the fluorescence intensity of the donor and acceptor FP can be utilized as an optical read out to determine ligand concentrations in the sensor environment [5-8]. The question, to which extent good sensors make use of changes, either in distance or in relative orientation of the involved FPs (or a combination of both), is still under debate $[7,9,10]$. In this respect, further methodical approaches to gain relevant structural details of sensor constructs are provided directly by smallangle X-ray scattering (SAXS) studies [11-13] complemented by rigid-body modelling and molecular dynamics simulations [14,15]. In addition to sensor platforms, which employ unimolecular conformational FRET sensors (i.e., a single sensing protein flanked by two FPs), further types of fluorescence-based sensors have recently been developed. These sensor types include fluorescence modulated single FP-based sensors, complementation sensors, cleavage FRET sensors, or dimerization-based sensors [3,16,17].

Furthermore, we performed a combined analysis to characterize ligand induced conformational changes of three different constructs of a recently developed FRET-based glucose biosensor [18]. These constructs are based at the glucose-galactose binding protein from Escherichia coli (MglB) flanked by two FPs and make use of the Venus flytrap principle [7]. Since the three constructs differ significantly in their FRET intensity signal change upon glucose binding (sensor sensitivity), we investigated the corresponding conformational changes with different complementary approaches. First, SAXS measurements with the sensor constructs under ligand-free and ligand-saturated conditions were performed. Furthermore, we made use of recently acquired single molecule FRET data [18] and additionally conducted coarse-grained molecular dynamics simulations on the sensor construct with the best sensing performance. The aim of this combined analysis was to investigate how structural arrangements of the FPs change upon ligand binding and how this change is correlated with the change of the FRET transfer efficiency.

\section{Materials and Methods}

\subsection{Sensor Constructs}

Based on the FLII ${ }^{12} \mathrm{P}$-glu $600 \mu$ construct by Frommer and co-workers [7], we recently generated a sensor toolbox with different linker sequences flanking the central glucosegalactose binding protein from E. coli (MglB) and mTurquoise2 and Venus as the FRET pair [19]; see also Supplementary Materials Section S1. In this study, sensor constructs no. 1 and 4 were used as representatives for weak or only moderate sensor performance, while construct no. 2 represents a very efficient sensor [18]. For details about the chosen constructs, see Supplementary Materials, Figure S1.

\subsection{SAXS Measurements and Data Analysis}

Small-angle X-ray scattering (SAXS) experiments were performed on glucose sensor constructs, which were synthesized and purified as described earlier $[18,19]$. Since the glucose sensor constructs tend to form aggregates when concentrated to a $\mathrm{mg} / \mathrm{mL}$ range, a size exclusion chromatography (SEC)-SAXS approach was employed in the present study [20]. After the SEC column (Superdex 200 Increase 10/300 GL) was equilibrated with buffer ( $20 \mathrm{mM}$ MOPS, $150 \mathrm{mM} \mathrm{NaCl}$ in $\mathrm{H}_{2} \mathrm{O}, \mathrm{pH} 7.3$ ), $100 \mu \mathrm{L}$ of the respective glucose sensor construct was added to the column at a concentration of $5 \mathrm{mg} / \mathrm{mL}$ and $10 \mathrm{mg} / \mathrm{mL}$ via a $100 \mu \mathrm{L}$ loop. The three sensor constructs (nos. 1,2,4) were measured in the absence of glucose and under glucose saturated conditions (100 mM glucose in the buffer). Samples were diluted by a factor of around 1.4 during the SEC run. SAXS measurements were 
performed at the BM29 BIOSAXS beamline (ESRF, Grenoble, France) using a wavelength of $0.992 \AA$ [21]. SAXS data were recorded continuously during the SEC run and binned into data frames with a $1 \mathrm{~s}$ exposure time per frame. We used an automated data analysis pipeline protocol to select the corresponding X-ray frames of the monomeric SEC elution peak and of the pure buffer scattering. Data frames were automatically checked for the absence of protein aggregation, for radiation damage and merged. Buffer scattering was subtracted from the merged data frames and buffer-subtracted data was normalized by the measured protein concentration. Finally, the normalized SAXS data of the $5 \mathrm{mg} / \mathrm{mL}$ and $10 \mathrm{mg} / \mathrm{mL}$ protein solutions were compared and no effects due to protein-protein interactions were found. Therefore, normalized SAXS data of both concentrations were merged and used for further data analysis. Temperature at the sample position was $293 \mathrm{~K}$ during the experiment, the used $\mathrm{X}$-ray detector was a Pilatus $1 \mathrm{M}$ and sample to detector distance was $2.869 \mathrm{~m}$. Further data analysis was performed using the algorithms available within the ATSAS software package [22]. The distance distribution function $P(r)$ and the Porod volume of the protein were determined with the programs, GNOM and DATPOROD, respectively. Guinier analysis was performed in a $q$-range with $q R_{\mathrm{G}}<1.3$ yielding the Guinier radius $R_{\mathrm{G}}$ and the forward scattering $I(0)$. Maximal dimension of the particle $D_{\max }$ and Guinier radii in real $R_{\mathrm{G} \text {,real }}$ and reciprocal space $R_{\mathrm{G} \text {,rec }}$ were determined from the $P(r)$ function. The molecular weight was calculated from the Porod volume as described by Petoukhov et al. [23] using a proportionality factor of 0.625 . In addition, the molecular mass was also determined using Bayesian inference based on SAXS different invariants as described by Hajizadeh et al. [24].

\subsection{Modelling and Simulations}

We simulated sensor construct no. 2 with the simulation framework of structurebased models (SBMs) [25], implemented in eSBMTools [26] to accommodate for large-scale dynamics and significant time scales. Based on energy landscape theory and the principle of minimal frustration, SMBs are a coarse-grained MD variant widely applied for either large, long, or both, timescale simulations [27]. For the simulations, a starting structure of atomic detail as well as parameters for the system were required. Based on the sensor sequence (see Supplementary Materials, Section S1) we first modeled a structure of the sensor construct by attaching the different structural parts successively. For the glucose/galactose binding protein (MglB) and the FPs we used the respective PDB structures (MglB: 2FVY and 2FW0 [4], mTurquoise2: 3ZTF [28], Venus: 1MYW [29]). Since there is no determined three-dimensional structure for the linkers, only limited information about their structural and dynamic properties was available. To incorporate the linkers into the simulations, we generated structures based on their sequence. This generation of structures resulted in a wide ensemble of possible structures for the sensor construct. To narrow the conformational space for the simulations, we fitted the resulting structures to the respective experimental SAXS data of the sensor in the glucose bound state using CRYSOL [30]. As a starting point for the simulations, we chose the four best fitting structures with the lowest $\chi^{2}$ values (see Section 3.2). We parametrized proteins and linkers similar to dye-labeled proteins [31]. All simulations of the sensor construct were performed with GROMACS v4.5.4 [32] with the extension for Gaussian contact potentials [31], Langevin dynamics and an SBM potential; see $[31,33]$. In the simulations, the linker was left rather flexible in comparison to the rest of the structure, to account for its flexibility and the uncertainty regarding its structure. As SBMs do not have an inherent time scale, we determined the time scale by comparison of rotational correlation times of free FPs between simulations and experimental values. For the evaluation, we generated SAXS intensity profiles of structures distributed evenly over the entire simulation using CRYSOL $[30,34]$. To compare the rotational flexibility of both FPs in the sensor, we calculated the fluorescence anisotropy and the rotational correlation times of the FPs bound to MglB. By fitting the simulated trajectory of MglB, we could observe the FP motions independently of the overall rotation of the system, which is experimentally hard to achieve. Due to the restriction of the rotational motions, 
we used the wobbling-in-a-cone model, where the anisotropy decay is described by the rotational correlation time, the fundamental anisotropy and the angle of the cone [35]. More details about the structures, parameters, simulation procedure and analysis are given in the Supplementary Materials, Section S2.

\section{Results and Discussion}

\subsection{Comparison of Single Molecule FRET and SAXS Results}

The sensor constructs used here were identified from a previously developed sensor toolbox $[18,19]$. As already stated by Deuschle and co-workers, a good sensor design is closely related to establishing a strong allosteric linkage between the ligand-induced conformational change in the glucose/galactose binding protein $(\mathrm{MglB})$ and the chromophore rearrangement [7]. In that respect, short linkers connecting the MglB with each of the FPs must be chosen in a way that (i) the distance between both FPs remains close enough to observe FRET changes and (ii) the rotational freedom of one or both FPs should be reduced in order to obtain a better coupling of conformational change to FRET change. Therefore, the sensors were constructed with the donor FP inserted between positions 12 and 13 at the N-terminal end of the MglB sequence, thereby establishing a tighter link of the donor FP to the binding region [7].

In addition to the sensor prototype without linkers (no. 1), one construct with a short flexible linker at the N-terminal FP (no. 2) and another one with a flexible linker at the C-terminal FP (no. 4) were characterized by SAXS measurements (for corresponding sequences see Supplementary Materials, Section S1). The respective individual sensor sensitivities, measured by the FRET signal changes for these constructs, were characterized in detail earlier [18] and the corresponding results are summarized in Figure 1 and Table 1. The sensitivity of a sensor construct is determined by measuring the ratio of acceptor and donor fluorescence emission $\left(\mathrm{R}=\mathrm{I}_{\mathrm{A}} / \mathrm{I}_{\mathrm{D}}\right)$ as a function of ligand (glucose) concentration. Effectively, the obtained R-value is a measure of the Förster resonance energy transfer which changes upon ligand binding. The resulting sigmoidal shaped binding isotherms yield the corresponding apparent dissociation constant and the effective sensitivity of the sensor construct, given by $\Delta \mathrm{R}$ (see [18]). The latter displays the difference between $\mathrm{R}$-values in absence of the ligand $\left(R_{\min }\right)$ and $R$-values saturated with ligand $\left(R_{\max }\right)$. The $K_{d}$-values for D-glucose of the investigated constructs range between a few and a few tens of $\mathrm{mM}$, while above $100 \mathrm{mM}$ all constructs are fully saturated with glucose.

Table 1. Comparison of structural parameters for the different sensor constructs.

\begin{tabular}{|c|c|c|c|c|c|c|}
\hline & No. 1 & $\begin{array}{c}\text { No. } 1 \\
+ \text { Glucose }\end{array}$ & No. 2 & $\begin{array}{l}\text { No. } 2 \\
+ \text { Glucose }\end{array}$ & No. 4 & $\begin{aligned} & \text { No. } 4 \\
+ & \text { Glucose }\end{aligned}$ \\
\hline $\begin{array}{l}\mathrm{R}_{\mathrm{G}}(\AA) \\
\text { Guinier }\end{array}$ & $\begin{array}{l}38.87 \\
\pm 0.06\end{array}$ & $\begin{array}{l}38.60 \\
\pm 0.08\end{array}$ & $\begin{array}{l}40.25 \\
\pm 0.11\end{array}$ & $\begin{array}{c}37.91 \\
\pm 0.07\end{array}$ & $\begin{array}{c}40.42 \\
\pm 0.10\end{array}$ & $\begin{array}{c}40.05 \\
\pm 0.08\end{array}$ \\
\hline$<\mathrm{E}>1$ & $\begin{array}{c}0.624 \\
\pm 0.023 \\
\end{array}$ & $\begin{array}{c}0.690 \\
\pm 0.020 \\
\end{array}$ & $\begin{array}{c}0.129 \\
\pm 0.040 \\
\end{array}$ & $\begin{array}{c}0.596 \\
\pm 0.040 \\
\end{array}$ & $\begin{array}{c}0.289 \\
\pm 0.040 \\
\end{array}$ & $\begin{array}{c}0.472 \\
\pm 0.040\end{array}$ \\
\hline$\Delta \mathrm{R}_{\mathrm{G}}(\AA)^{2}$ & \multicolumn{2}{|c|}{$0.27 \pm 0.14$} & \multicolumn{2}{|c|}{$2.34 \pm 0.18$} & \multicolumn{2}{|c|}{$0.37 \pm 0.18$} \\
\hline$\Delta \mathrm{E}^{3}$ & \multicolumn{2}{|c|}{$0.066 \pm 0.043$} & \multicolumn{2}{|c|}{$0.467 \pm 0.08$} & \multicolumn{2}{|c|}{$0.183 \pm 0.08$} \\
\hline$\Delta \mathrm{E} / \Delta \mathrm{R}_{\mathrm{G}}\left(\AA^{-1}\right)$ & \multicolumn{2}{|c|}{0.244} & \multicolumn{2}{|c|}{0.199} & \multicolumn{2}{|c|}{0.459} \\
\hline$\Delta \mathrm{R}$ & \multicolumn{2}{|c|}{0.40} & \multicolumn{2}{|c|}{0.98} & \multicolumn{2}{|c|}{0.22} \\
\hline
\end{tabular}

${ }^{1}$ Obtained mean position of the individual population in the smFRET histogram (see Figure 1a). ${ }^{2}$ Difference between glucose-saturated and glucose-free states calculated based on the $\mathrm{R}_{\mathrm{G}}$ values. ${ }^{3}$ Shift of the weighted mean position of the glucose-bound and glucose-free populations as calculated in a previous publication; see [18]. 

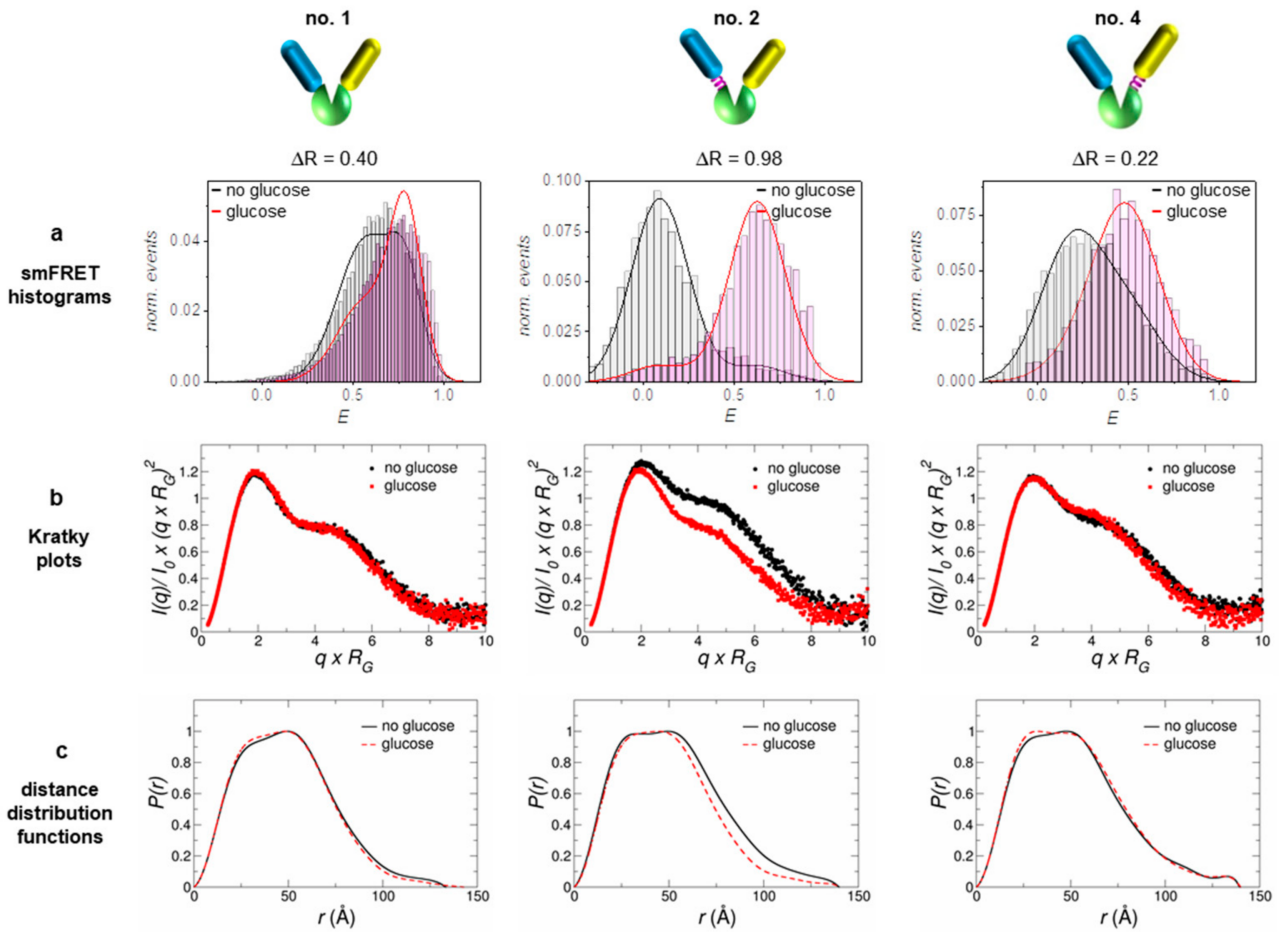

Figure 1. The three investigated sensor constructs exhibit structural differences with respect to how the FPs are attached to the glucose binding protein MglB: no linker between MglB and both FPs (no. 1, left), a flexible linker between MglB and the donor FP (no. 2, middle) and a flexible linker between MglB and the acceptor FP (no. 4, right). The corresponding $\Delta \mathrm{R}$-values were determined from ensemble Förster resonance energy transfer (FRET) measurements (FRET binding isotherms, see [18]). (a) Data from single molecule measurements (smFRET histograms) were taken from an earlier publication, where these constructs were analyzed in detail [18]. Based on the analysis of the measured small angle X-ray scattering (SAXS) data, the respective Kratky plots (b) and distance distribution functions (c) are presented in the two lower lines. For further details, refer to the Supplementary Materials, Figures S3.2 and S3.3, and Table S2.

In order to obtain a more detailed picture about how a reasonable sensor sensitivity is achieved, we used single molecule FRET data from earlier measurements; for details see [18]. In accordance with the obtained $\Delta \mathrm{R}$-values, the population shift $\Delta \mathrm{E}$ is most pronounced in the case of sensor no. 2 (see Figure 1a and Table 1). Only for construct no. 2 we observed two well-separated populations for each state, with an almost complete $(\sim 90 \%)$ transfer from one state to the other upon ligand binding/release. Compared to this, the other two constructs show a significantly smaller shift between the populations, where that of construct no. 1 is still smaller when compared to that of construct no. 4 (see Table 1).

To obtain direct structural information on the sensor constructs, we performed SAXS measurements with the constructs in the glucose-bound state (displayed in red) and the glucose-free state (displayed in black); see Figure 1b,c. The Kratky plots as well as the distance distribution functions clearly discriminate construct no. 2 from the other two. Only sensor no. 2 displays a pronounced structural compaction upon glucose binding in the respective plots. This fact is also supported by the obtained $R_{G}$-values from a Guinier analysis given in Table 1.

Nevertheless, the other sensor constructs also exhibit a small compaction upon ligand binding, although smaller by a factor of 6-9 (in terms of $\Delta R_{G}$ ) as compared to construct no. 2 . Qualitatively, all sensor constructs follow the same structural behavior of the pure glucose binding protein $\mathrm{MglB}$, which also shows a distinct compaction upon glucose binding (see Supplementary Materials, Figure S3.1). Although the radius of gyration $R_{G}$ is by definition not equal to the distance $R_{D A}$ between both FPs (relevant FRET parameter), the change in $R_{G}\left(\Delta R_{G}\right)$ and the change in energy transfer $\Delta E$ appear to be highly correlated (see Table 1 
and Figure 2). Both corresponding difference values are the largest for sensor construct no. 2, followed by construct no. 4 , while construct no. 1 displays the smallest difference. Without knowing the relative orientation of the involved FPs (i.e., the respective transition dipole moments; see for example [36]), a direct measure of the inter-FP-distance change $\Delta \mathrm{R}_{\mathrm{DA}}$ upon substrate binding is not achievable. However, by looking at the $\Delta \mathrm{E} / \Delta \mathrm{R}_{\mathrm{G}}$ ratio (slope of solid lines in Figure 2) we can estimate to which extent the individual sensor construct makes use of a sensor compaction in order to achieve a certain change in transfer efficiency (i.e., sensor read out signal).

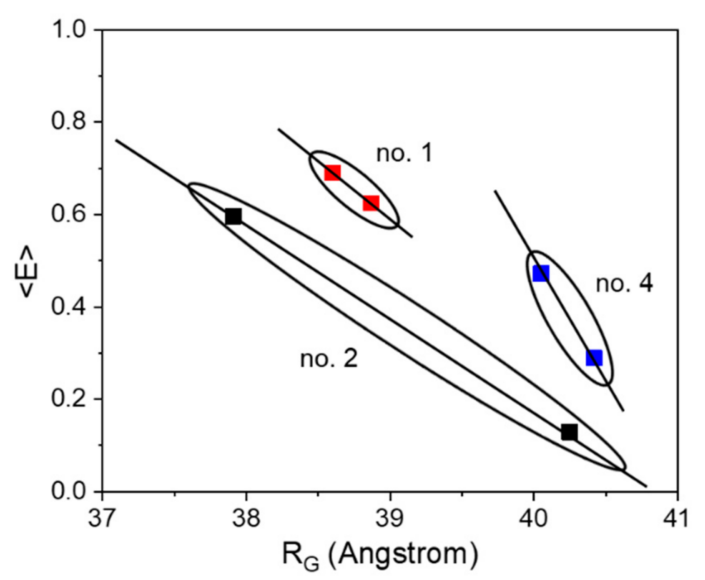

Figure 2. The correlation between energy transfer change and change in $R_{G}$ is shown for the three investigated sensor constructs. Although we do not expect a strict linear relationship between $<\mathrm{E}>$ and $R_{G}$, the solid lines do not represent fits, but should visualize a general trend.

\subsection{Modelling and Coarse-Grained Molecular Dynamics Simulations of Sensor Construct no. 2}

To obtain a more detailed understanding about the working principles of the FRET sensor constructs on a molecular level, rigid-body modelling and coarse-grained molecular dynamics simulations were performed for construct no. 2. Since the latter exhibits the highest sensor sensitivity in terms of FRET signal change, we chose this construct for simulation studies. For this purpose, we first prepared rigid body models with two distinct MglB crystal structures, one with ligand bound and one without. The comparison of both structures' stresses exhibits a significant conformational change upon glucose binding which is characterized by a pronounced hinge-twist motion of the two domains against each other [4]. Due to the ability to transfer the MglB conformational change into a large FRETratio change, the flexible linker between MglB and the donor FP of the sensor construct no. 2 appears to be crucial for the superior performance of this sensor (see sequence of this construct in Figure 3a).

The merging of the sensor construct no. 2 resulted in a wide ensemble of possible structures. We fitted the resulting structures to experimental SAXS data and chose four different starting structures for the glucose-bound state (see Figure 3c) which were selected based on the lowest $\chi^{2}$-values (see Figure $3 b$ and legend of Figure 3). Further simulations with these different starting structures were performed for the glucose-saturated and in the glucose-free state. Each simulation represents the dynamics of the system during approximately $100 \mu \mathrm{s}$ on the physical time scale (for methodical details of the simulation see Supplementary Materials, Section S2).

For the glucose-bound state of one sensor no. 2 starting structure (orange FPs in Figure 3c, orange in Figure 3b) we exemplarily show a comparison of the simulated data with experimental SAXS data. The approach of using different starting structures in our simulations helped to cover the large conformational space of the flexible sensor constructs. Structures from the simulations showing the best concordance with the experimental SAXS data are shown in green in Figure 3b. In the case of the ligand-saturated state, this is very similar to the starting structure (Figure $3 \mathrm{~b}$, orange fit). The obtained $\chi^{2}$ values describing 
the deviation of the mean intensity curve of the simulation with respect to the experimental SAXS data are quite small, for the glucose-free state (lower panel in Figure 3b) as well as for the glucose-saturated state (upper panel in Figure 3b).

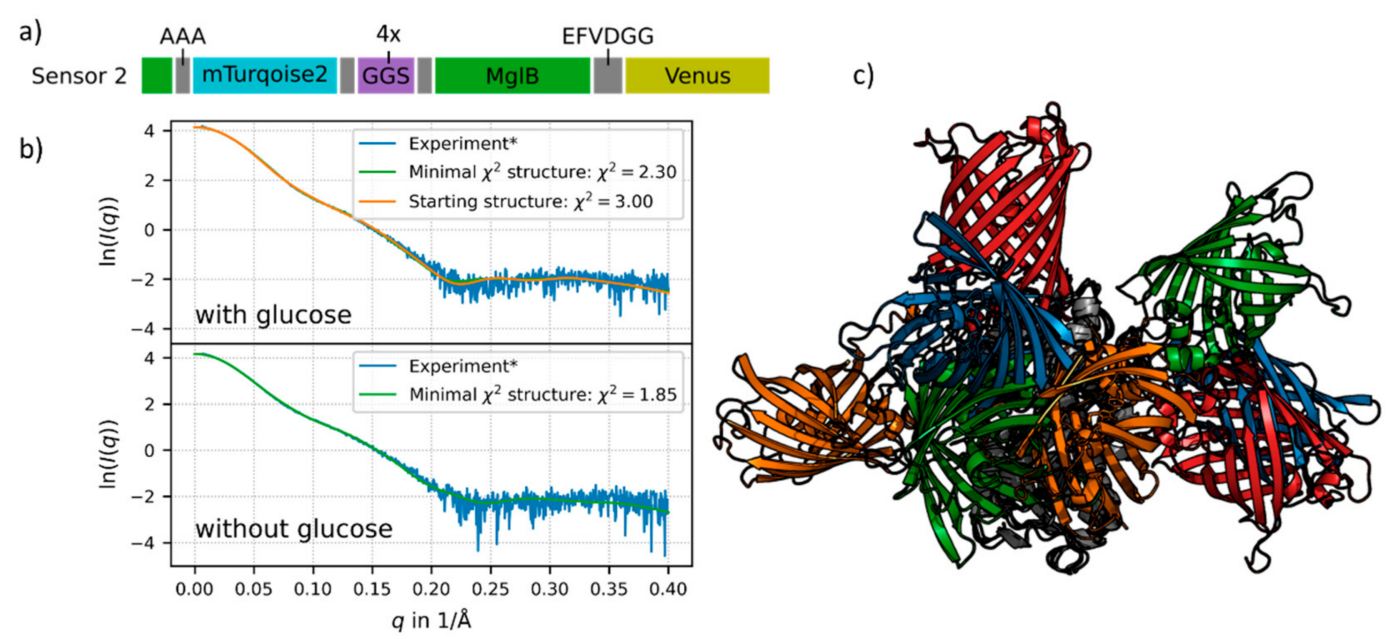

Figure 3. (a) Schematic presentation of the sensor no. 2 sequence. The flexible linker (purple) and restriction sites (grey) are shown with their respective sequences. The donor FP (mTurquoise2, cyan) is inserted into MglB (green), so the first 11 residues of MglB are in the beginning of the sequence and the main part in the middle. The acceptor FP (Venus) is shown in yellow (for details see Supplementary Materials, Section S1). (b) The experimental SAXS data is shown in blue, the fit of the chosen starting structure (blue FPs in Figure 3c) in orange, the latter is only shown for the glucose-saturated structure. Structures from the simulations with respective minimal $\chi^{2}$ values are shown in green. (c) Starting structures for the simulation of sensor no. 2 with MglB in the glucose-saturated state. All structures are aligned to MglB (grey). The different pairs of fluorescent proteins are depicted in orange (sensor $2-1, \chi^{2}=3.0$ ), blue (sensor $2-2, \chi^{2}=3.11$ ), green (sensor 2-3, $\chi^{2}=3.75$ ) and red (sensor $2-4, \chi^{2}=4.1$ ). For details see Supplementary Materials, Section S2.6.

The simulation trajectories were then utilized to analyze the rotational mobility of each of the attached FPs (for details see Supplementary Materials, Section S2.9). For this purpose, we calculated the time-dependent fluorescence anisotropy and the rotational correlation times of the chromophores (as part of the FPs) bound to MglB. This approach allowed obtaining the motion of the fluorophore independently of the overall sensor construct rotation, in contrast to the experimental time resolved anisotropy measurements. Two exemplary fits are shown in Figure 4 a. Due to possible restrictions of the FP motion, the "wobbling-in-a-cone" model [35] is more appropriate here than a simple exponential fit. This type of analysis is well suited to determine approximations of rotational correlation times of the fluorescent proteins in the sensor, as they are not accessible directly by the conducted experiments. The derived values for rotational correlation times for the donor FP and the acceptor FP in the sensor construct are on a physical time scale of 190-290 ns.

This indicates that the FPs are still mobile, but on a much longer time scale as compared to, for example, free fluorescent protein (correlation time of about $20 \mathrm{~ns}$ ). The rotational correlation times of the attached fluorophores are also much longer than the fluorescence lifetimes of both fluorophores (a few ns). Therefore, the normally applied approach of isotropic dynamic averaging (resulting in a $\kappa^{2}$-value of $2 / 3$ ) cannot be applied. Importantly, these results support the assumption that for improved sensor design, the rotational freedom of at least one FP should be reduced. Based on our results, the rotational mobility of the inserted donor FP at the N-terminal end of construct no. 2 is geometrically hindered. This conclusion is supported by a relatively large amplitude factor A (or large $r(t)$ at long $\left.\Delta \mathrm{t}_{\mathrm{SBM}}\right)$ as shown for the ligand bound state in Figure $4 \mathrm{a}$. Due to this non-isotropic donor FP rotation in the ligand-bound state, we could not calculate reliable $\kappa^{2}$ and $R_{D A}$ values from 
the experimental FRET data, which excludes a direct comparison between experimentally determined and simulated inter FP distances in the present. In contrast, the acceptor FP is fused directly to the C-terminal end of $\mathrm{MglB}$ and does not show such restrictions (see small A-value in Figure 4a). Additionally, the donor FP regains flexibility, i.e., a decrease in A-values, in structures without glucose, whereas this change is negligible for the acceptor FP (see Supplementary Materials, Section S2.9).
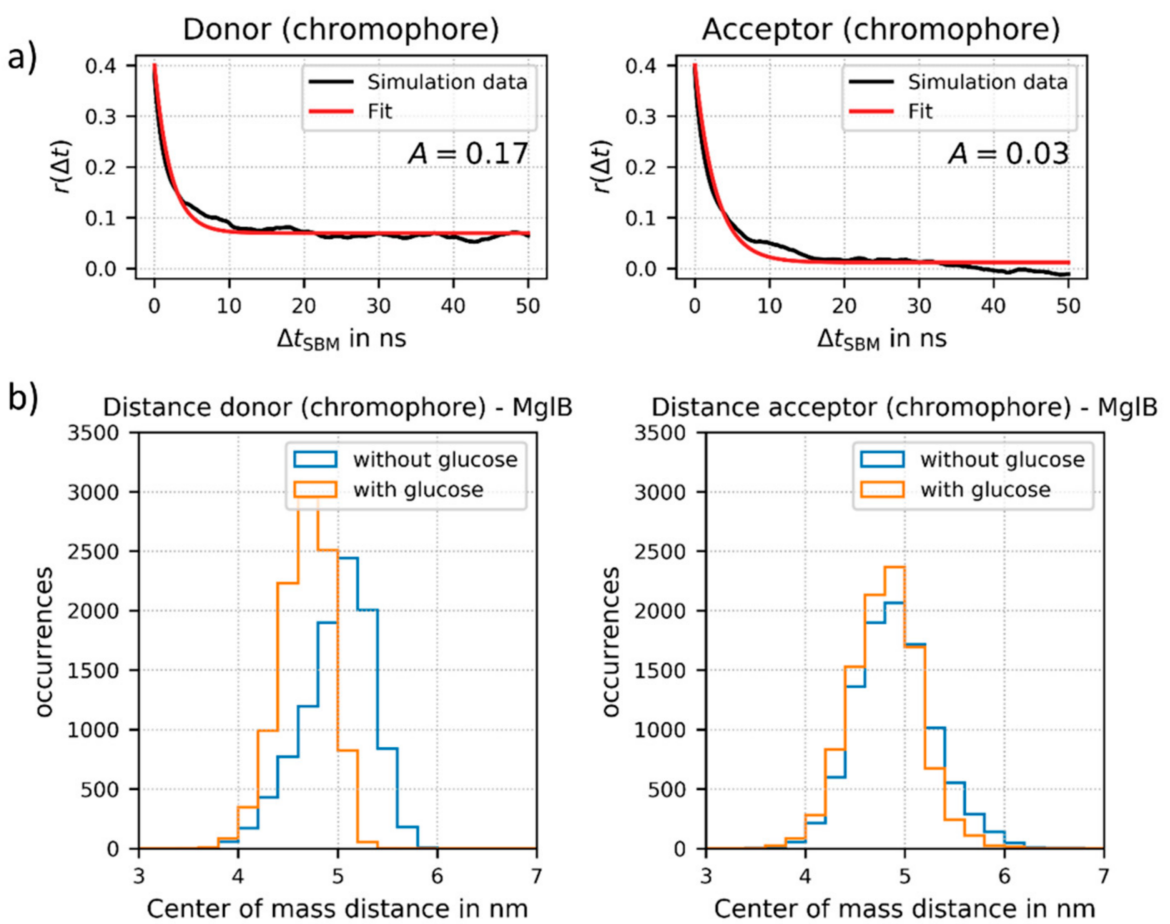

Figure 4. (a) Fits of the fluorescence anisotropy $r(\Delta t)$ as a function of time $\Delta t_{\text {SBM }}$ for the donor chromophore (as part of mTurquoise2) and for the acceptor chromophore (as part of Venus) attached to $\mathrm{MglB}$ (here in the glucose-bound state). The time $\Delta \mathrm{t}_{\mathrm{SBM}}$ is given in ns in the SBM simulation, which does not directly correspond to a physical time scale. The model function for the "wobbling-in-a cone" model (solid red) was fitted to $r(\Delta t)$ values obtained from the simulation of sensor no. 2 . The respective rotational correlation times on a physical time scale are in a time regime of 190-290 ns (further details see Supplementary Materials, Section S2). (b) Relative distance change upon glucose binding between the donor FP and the center of the MglB (left) and between the acceptor FP and the $\mathrm{MglB}$ (right), respectively. For the distance calculation we used the center of mass of the respective chromophore of the FPs.

Finally, we also analyzed the changes in distance between the donor FP and the center of the MglB structure and between the acceptor FP and the MglB, respectively. We could demonstrate that during the simulations the distance between the donor FP and MglB displays a considerable change upon glucose binding (see Figure $4 \mathrm{~b}$ ). Therefore, our simulations further support an important prerequisite of the ideal sensor design, a strong allosteric coupling achieved by inserting the donor FP after position 12 at the $\mathrm{N}$-terminal end of the MglB sequence (see Supplementary Materials, Section S1). In contrast, the distance between the acceptor FP and MglB is not altered significantly upon glucose binding.

\section{Conclusions}

One key in FRET-based biosensor development is to understand and to make use of the structure-function relationship of the sensor constructs which are typically artificial man-made multi-modular fusion proteins. Therefore, complementary to the required FRET measurements, additional methodical approaches like SAXS, NMR, X-ray crystallography 
and rigid body modelling were already employed in the past to unravel the structurefunction relationship $[9,11,12,37]$. In our approach, we additionally propose the use of single molecule FRET measurements, SAXS experiments and coarse-grained molecular dynamics simulations [31,38].

As demonstrated by the presented SAXS measurements, only a sensor with pronounced conformational change upon glucose binding (sensor no. 2) shows a large change in FRET efficiency which is necessary for a high sensitivity. Although the other two analyzed constructs (no. 1 and no. 4) experienced a similar ligand-induced conformational change of the central binding protein, the resulting conformational changes of the corresponding whole constructs were much less pronounced. As a consequence, the constructs no. 1 and no. 4 show a much smaller, but still measurable, FRET signal change. In summary, we can conclude that only a pronounced, ligand-induced conformational change leads to a considerable sensor sensitivity.

These findings were supported by coarse-grained molecular dynamics simulations. Even though simulations of sensor no. 2 showed relatively large conformational flexibility for the ligand-bound and ligand-free state, sampled within a $100 \mu$ s time window, we obtained evidence for a pronounced difference in the mean inter FP distance between the ligand-free and the ligand bound-state (see Supplementary Materials, Figure S2.6). Furthermore, we obtained donor FP-MglB distances values for sensor no. 2 that also show a considerable difference between the ligand-free and ligand-bound state.

Despite this flexibility, the simulation also revealed a reduced rotational mobility of the donor FP due to spatial constrains such as its restricted distance to MglB. In contrast, the acceptor mobility is not hindered. All observed changes between both states are clearly supported by the SAXS data. Our example indicates that, for a good FRET-based sensor, a tight allosteric coupling to at least one of the two attached FPs is sufficient.

Supplementary Materials: The following are available online at https:/ / www.mdpi.com/article/ 10.3390/s21124144/s1, Documents S1. Sensor constructs and experimental sequences; S2. Simulations of biosensors with fluorescent proteins; S3. SAXS results; Figures S1.1, S2.1-S2.8, S3.1-S3.3; Tables S1 and S2 (PDF).

Author Contributions: J.F., M.P., A.M.S. and A.S. conceived the idea and designed the experiments; J.O. and M.S. produced, characterized the samples and performed the SAXS measurements and M.S. evaluated the SAXS data; I.R. performed the modelling and MD simulations; I.R., H.H., and A.M.S. compiled the interpretation of experimental and simulation results; J.F., I.R. and J.O. wrote the manuscript with input from all authors. All authors have read and agreed to the published version of the manuscript.

Funding: The simulations in this work were performed on the supercomputer ForHLR funded by the Ministry of Science, Research and the Arts Baden-Württemberg and by the Federal Ministry of Education and Research. H.H. thanks the International Helmholtz Research School on Biophysics and Soft Matter (BioSoft) for financial support. J.F., A.M.S. and M.S. acknowledge funding by the BMBF project 05K16PA1. The present contribution was supported by the Helmholtz Association under the joint research school "HIDSS4Health-Helmholtz Information and Data Science School for Health".

Institutional Review Board Statement: Not applicable.

Informed Consent Statement: Not applicable.

Data Availability Statement: Not applicable.

Acknowledgments: We acknowledge the European Synchrotron Radiation Facility (ESRF) for provision of synchrotron radiation facilities and thank Mark Tully for assistance in using beamline BM29.

Conflicts of Interest: The authors declare no conflict of interest. 


\section{References}

1. Lindenburg, L.; Merkx, M. Engineering Genetically Encoded FRET Sensors. Sensors 2014, 14, 11691-11713. [CrossRef]

2. Hochreiter, B.; Garcia, A.P.; Schmid, J.A. Fluorescent proteins as genetically encoded FRET biosensors in life sciences. Sensors 2015, 15, 26281-26314. [CrossRef] [PubMed]

3. Sanford, L.; Palmer, A. Recent Advances in Development of Genetically Encoded Fluorescent Sensors. Methods Enzymol. 2017, 589, 1-49. [CrossRef] [PubMed]

4. Borrok, M.J.; Kiessling, L.L.; Forest, K.T. Conformational changes of glucose/galactose-binding protein illuminated by open, unliganded, and ultra-high-resolution ligand-bound structures. Protein Sci. 2007, 16, 1032-1041. [CrossRef]

5. Dwyer, M.A.; Hellinga, H.W. Periplasmic binding proteins: A versatile superfamily for protein engineering. Curr. Opin. Struct. Biol. 2004, 14, 495-504. [CrossRef]

6. De Lorimier, R.M.; Smith, J.J.; Dwyer, M.A.; Looger, L.L.; Sali, K.M.; Paavola, C.D.; Rizk, S.S.; Sadigov, S.; Conrad, D.W.; Loew, L.; et al. Construction of a fluorescent biosensor family. Protein Sci. 2009, 11, 2655-2675. [CrossRef] [PubMed]

7. Deuschle, K.; Okumoto, S.; Fehr, M.; Looger, L.L.; Kozhukh, L.; Frommer, W.B. Construction and optimization of a family of genetically encoded metabolite sensors by semirational protein engineering. Protein Sci. 2005, 14, 2304-2314. [CrossRef]

8. Merkx, M.; Smith, B.; Jewett, M. Engineering Sensor Proteins. ACS Sensors 2019, 4, 3089-3091. [CrossRef] [PubMed]

9. Pham, E.; Chiang, J.; Li, I.; Shum, W.; Truong, K. A Computational Tool for Designing FRET Protein Biosensors by Rigid-Body Sampling of Their Conformational Space. Structure 2007, 15, 515-523. [CrossRef]

10. Campbell, R.E. Fluorescent-Protein-Based Biosensors: Modulation of Energy Transfer as a Design Principle. Anal. Chem. 2009, 81, 5972-5979. [CrossRef]

11. Mertens, H.D.; Piljić, A.; Schultz, C.; Svergun, D.I. Conformational Analysis of a Genetically Encoded FRET Biosensor by SAXS. Biophys. J. 2012, 102, 2866-2875. [CrossRef] [PubMed]

12. Geiger, A.; Russo, L.; Gensch, T.; Thestrup, T.; Becker, S.; Hopfner, K.P.; Griesinger, C.; Witte, G.; Griesbeck, O. Correlating Calcium Binding, Forster Resonance Energy Transfer, and Conformational Change in the Biosensor TN-XXL. Biophys. J. 2012, 102, 2401-2410. [CrossRef] [PubMed]

13. Faccio, G.; Salentinig, S. Enzyme-Triggered Dissociation of a FRET-Based Protein Biosensor Monitored by Synchrotron SAXS. Biophys. J. 2017, 113, 1731-1737. [CrossRef]

14. Gerstein, M.; Lesk, A.M.; Chothia, C. Structural Mechanisms for Domain Movements in Proteins. Biochemical 1994, 33, 6739-6749. [CrossRef]

15. Hinsen, K.; Thomas, A.; Field, M.J. Analysis of domain motions in large proteins. Proteins Struct. Funct. Bioinform. 1999, 34, 369-382. [CrossRef]

16. Vinkenborg, J.L.; Evers, T.H.; Reulen, S.W.A.; Meijer, E.W.; Merkx, M. Enhanced sensitivity of FRET-based protease sensors by redesign of the GFP dimerization interface. ChemBioChem 2007, 8, 1119-1121. [CrossRef] [PubMed]

17. Tamura, T.; Hamachi, I. Recent Progress in Design of Protein-Based Fluorescent Biosensors and Their Cellular Applications. ACS Chem. Biol. 2014, 9, 2708-2717. [CrossRef]

18. Höfig, H.; Otten, J.; Steffen, V.; Pohl, M.; Boersma, A.J.; Fitter, J. Genetically Encoded Forster Resonance Energy Transfer-Based Biosensors Studied on the Single-Molecule Level. ACS Sens. 2018, 3, 1462-1470. [CrossRef] [PubMed]

19. Otten, J.; Tenhaef, N.; Jansen, R.P.; Döbber, J.; Jungbluth, L.; Noack, S.; Oldiges, M.; Wiechert, W.; Pohl, M. A FRET-based biosensor for the quantification of glucose in culture supernatants of $\mathrm{mL}$ scale microbial cultivations. Microb. Cell Factories 2019, 18, 1-10. [CrossRef] [PubMed]

20. Brennich, M.E.; Round, A.R.; Hutin, S. Online Size-exclusion and Ion-exchange Chromatography on a SAXS Beamline. J. Vis. Exp. 2017, e54861. [CrossRef]

21. Pernot, P.; Theveneau, P.; Giraud, T.; Fernandes, R.N.; Nurizzo, D.; Spruce, D.; Surr, J.; McSweeney, S.; Round, A.; Felisaz, F.; et al. New beamline dedicated to solution scattering from biological macromolecules at the ESRF. J. Phys. Conf. Ser. 2010, $247,247$. [CrossRef]

22. Franke, D.; Petoukhov, M.V.; Konarev, P.V.; Panjkovich, A.; Tuukkanen, A.; Mertens, H.D.T.; Kikhney, A.G.; Hajizadeh, N.R.; Franklin, J.M.; Jeffries, C.M.; et al. ATSAS 2.8: A comprehensive data analysis suite for small-angle scattering from macromolecular solutions. J. Appl. Crystallogr. 2017, 50, 1212-1225. [CrossRef]

23. Petoukhov, M.V.; Franke, D.; Shkumatov, A.V.; Tria, G.; Kikhney, A.G.; Gajda, M.; Gorba, C.; Mertens, H.D.T.; Konarev, P.V.; Svergun, D.I. New developments in the ATSAS program package for small-angle scattering data analysis. J. Appl. Crystallogr. 2012, 45, 342-350. [CrossRef]

24. Hajizadeh, N.R.; Franke, D.; Jeffries, C.M.; Svergun, D.I. Consensus Bayesian assessment of protein molecular mass from solution X-ray scattering data. Sci. Rep. 2018, 8, 1-13. [CrossRef]

25. Bryngelson, J.D.; Onuchic, J.N.; Socci, N.D.; Wolynes, P.G. Funnels, pathways, and the energy landscape of protein folding: A synthesis. Proteins 1995, 21, 167-195. [CrossRef] [PubMed]

26. Lutz, B.; Sinner, C.; Heuermann, G.; Verma, A.; Schug, A. eSBMTools 1.0: Enhanced native structure-based modeling tools. Bioinformation 2013, 29, 2795-2796. [CrossRef]

27. Onuchic, J.N.; Luthey-Schulten, Z.; Wolynes, P.G. THEORY OF PROTEIN FOLDING: The Energy Landscape Perspective. Annu. Rev. Phys. Chem. 1997, 48, 545-600. [CrossRef] [PubMed] 
28. Rekas, A.; Alattia, J.R.; Nagai, T.; Miyawaki, A.; Ikura, M. Crystal structure of venus, a yellow fluorescent protein with im-proved maturation and reduced environmental sensitivity. J. Biol. Chem. 2002, 277, 50573-50578. [CrossRef] [PubMed]

29. Goedhart, J.; Von Stetten, D.; Noirclerc-Savoye, M.; Lelimousin, M.; Joosen, L.; Hink, M.A.; Van Weeren, L.; Jr, T.W.G.; Royant, A. Structure-guided evolution of cyan fluorescent proteins towards a quantum yield of $93 \%$. Nat. Commun. 2012, 3, 751. [CrossRef] [PubMed]

30. Svergun, D.; Barberato, C.; Koch, M.H.J. CRYSOL-A program to evaluate x-ray solution scattering of biological macro-molecules from atomic coordinates. J. Appl. Crystallogr. 1995, 28, 768-773. [CrossRef]

31. Reinartz, I.; Sinner, C.; Nettels, D.; Stucki-Buchli, B.; Stockmar, F.; Panek, P.T.; Jacob, C.R.; Nienhaus, G.U.; Schuler, B.; Schug, A. Simulation of FRET dyes allows quantitative comparison against experimental data. J. Chem. Phys. 2018, 148, 123321. [CrossRef]

32. Van Der Spoel, D.; Lindahl, E.; Hess, B.; Groenhof, G.; Mark, A.E.; Berendsen, H.J.C. GROMACS: Fast, flexible, and free. J. Comput. Chem. 2005, 26, 1701-1718. [CrossRef] [PubMed]

33. Noel, J.K.; Schug, A.; Verma, A.; Wenzel, W.; Garcia, A.E.; Onuchic, J.N. Mirror Images as Naturally Competing Conformations in Protein Folding. J. Phys. Chem. B 2012, 116, 6880-6888. [CrossRef] [PubMed]

34. Reinartz, I.; Weiel, M.; Schug, A. FRET Dyes Significantly Affect SAXS Intensities of Proteins. Isr. J. Chem. 2020, 60, 725-734. [CrossRef]

35. Kinosita, K.; Kawato, S.; Ikegami, A. A theory of fluorescence polarization decay in membranes. Biophys. J. 1977, 20, 289-305. [CrossRef]

36. Förster, T. Zwischenmolekulare Energiewanderung und Fluoreszenz. Ann. Phys. 1948, 437, 55-75. [CrossRef]

37. Trigo-Mourino, P.; Thestrup, T.; Griesbeck, O.; Griesinger, C.; Becker, S. Dynamic tuning of FRET in a green fluorescent pro-tein biosensor. Sci. Adv. 2019, 5, eaaw4988. [CrossRef]

38. Gabba, M.; Poblete, S.; Rosenkranz, T.; Katranidis, A.; Kempe, D.; Züchner, T.; Winkler, R.G.; Gompper, G.; Fitter, J. Conformational State Distributions and Catalytically Relevant Dynamics of a Hinge-Bending Enzyme Studied by Single-Molecule FRET and a Coarse-Grained Simulation. Biophys. J. 2014, 107, 1913-1923. [CrossRef] 